\title{
Face Recognition Techniques: A Survey
}

\author{
V.Vijayakumari \\ Department of Electronics and Communication, Sri krishna College of Technology, Coimbatore, India \\ *Corresponding Author: ebinviji@rediffmail.com
}

Copyright $@ 2013$ Horizon Research Publishing All rights reserved.

\begin{abstract}
Face is the index of mind. It is a complex multidimensional structure and needs a good computing technique for recognition. While using automatic system for face recognition, computers are easily confused by changes in illumination, variation in poses and change in angles of faces. A numerous techniques are being used for security and authentication purposes which includes areas in detective agencies and military purpose. These surveys give the existing methods in automatic face recognition and formulate the way to still increase the performance.
\end{abstract}

Keywords Face Recognition, Illumination, Authentication, Security

\section{Introduction}

Developed in the 1960s, the first semi-automated system for face recognition required the administrator to locate features ( such as eyes, ears, nose, and mouth) on the photographs before it calculated distances and ratios to a common reference point, which were then compared to reference data. In the 1970s, Goldstein, Armon, and Lesk used 21 specific subjective markers such as hair color and lip thickness to automate the recognition. The problem with both of these early solutions was that the measurements and locations were manually computed. The face recognition problem can be divided into two main stages: face verification (or authentication), and face identification (or recognition). The detection stage is the first stage; it includes identifying and locating a face in an image. The recognition stage is the second stage; it includes feature extraction, where important information for the discrimination is saved and the matching where the recognition result is given aid of a face database.

\section{Methods}

\subsection{Geometric Feature Based Methods}

The geometric feature based approaches are the earliest approaches to face recognition and detection [1]. In these systems, the significant facial features are detected and the distances among them as well as other geometric characteristic are combined in a feature vector that is used to represent the face. To recognize a face, first the feature vector of the test image and of the image in the database is obtained. Second, a similarity measure between these vectors, most often a minimum distance criterion, is used to determine the identity of the face. As pointed out by Brunelli and Poggio, the template based approaches will outperform the early geometric feature based approaches [2].

\subsection{Template Based Methods}

The template based approaches represent the most popular technique used to recognize and detect faces [3]. Unlike the geometric feature based approaches, the template based approaches use a feature vector that represent the entire face template rather than the most significant facial features.

\subsection{Correlation Based Methods}

Correlation based methods for face detection are based on the computation of the normalized cross correlation coefficient $\mathrm{Cn}[4,5]$. The first step in these methods is to determine the location of the significant facial features such as eyes, nose or mouth. The importance of robust facial feature detection for both detection and recognition has resulted in the development of a variety of different facial feature detection algorithms. The facial feature detection method proposed by Brunelli and Poggio uses a set of templates to detect the position of the eyes in an image, by looking for the maximum absolute values of the normalized correlation coefficient of these templates at each point in test image $[6,2]$. To cope with scale variations, a set of templates at different scales was used.

The problems associated with the scale variations can be significantly reduced by using hierarchical correlation. For face recognition, the templates corresponding to the significant facial feature of the test images are compared in turn with the corresponding templates of all of the images in the database, returning a vector of matching scores computed through normalized cross correlation. The similarity scores of different features are integrated to obtain a global score that is used for recognition. Other similar method that use 
correlation or higher order statistics revealed the accuracy of these methods but also their complexity.

Beymer extended the correlation based on the approach to a view based approach for recognizing faces under varying orientation, including rotations with respect to the axis perpendicular to the image plane(rotations in image depth) [7]. To handle rotations out of the image plane, templates from different views were used. After the pose is determined ,the task of recognition is reduced to the classical correlation method in which the facial feature templates are matched to the corresponding templates of the appropriate view based models using the cross correlation coefficient. However this approach is highly computational expensive, and it is sensitive to lighting conditions.

\subsection{Matching Pursuit Based Methods}

Philips introduced a template based face detection and recognition system that uses a matching pursuit filter to obtain the face vector [8]. The matching pursuit algorithm applied to an image iteratively selects from a dictionary of basis functions the best decomposition of the image by minimizing the residue of the image in all iterations. The algorithm describes by Philips constructs the best decomposition of a set of images by iteratively optimizing a cost function, which is determined from the residues of the individual images [9]. The dictionary of basis functions used by the author consists of two dimensional wavelets, which gives a better image representation than the PCA (Principal Component Analysis) and LDA(Linear Discriminant Analysis) based techniques where the images were stored as vectors. For recognition the cost function is a measure of distances between faces and is maximized at each iteration. For detection the goal is to find a filter that clusters together in similar templates (the mean for example), and minimized in each iteration. The feature represents the average value of the projection of the templates on the selected basis

\subsection{Singular Value Decomposition Based Methods}

The face recognition method in this section use the general result stated by the singular value decomposition theorem. Z.Hong revealed the importance of using Singular Value Decomposition Method (SVD) for human face recognition by providing several important properties of the singular values (SV) vector which include: the stability of the SV vector to small perturbations caused by stochastic variation in the intensity image, the proportional variation of the SV vector with the pixel intensities, the variances of the SV feature vector to rotation, translation and mirror transformation [10]. The above properties of the SV vector provide the theoretical basis for using singular values as image features. In addition, it has been shown[11,2] that compressing the original SV vector into the low dimensional space by means of various mathematical transforms leads to the higher recognition performance. Among the various dimensionality reducing transformations, the Linear
Discriminant Transform is the most popular one.

\subsection{The Dynamic Link Matching Methods}

The above template based matching methods use an Euclidean distance to identify a face in a gallery or to detect a face from a background. A more flexible distance measure that accounts for common facial transformations is the dynamic link introduced by Lades et al.[7]. In this approach, a rectangular grid is centered all faces in the gallery. The feature vector is calculated based on Gabor type wavelets, computed at all points of the grid. A new face is identified if the cost function, which is a weighted sum of two terms, is minimized. The first term in the cost function is small when the distance between feature vectors is small and the second term is small when the relative distance between the grid points in the test and the gallery image is preserved. It is the second term of this cost function that gives the "elasticity" of this matching measure. While the grid of the image remains rectangular, the grid that is "best fit" over the test image is stretched. Under certain constraints, until the minimum of the cost function is achieved. The minimum value of the cost function is used further to identify the unknown face.

\subsection{Illumination Invariant Processing Methods}

The problem of determining functions of an image of an object that are insensitive to illumination changes are considered [12, 13]. An object with Lambertian reflection has no discriminative functions that are invariant to illumination. This result leads the author to adopt a probabilistic approach in which they analytically determine a probability distribution for the image gradient as a function of the surfaces geometry and reflectance. Their distribution reveals that the direction of the image gradient is insensitive to changes in illumination direction. Verify this empirically by constructing a distribution for the image gradient from more than twenty million samples of gradients in a database of thousand two hundred and eighty images of twenty inanimate objects taken under varying lighting conditions[14]. Using this distribution, they develop an illumination insensitive measure of image comparison and test it on the problem of face recognition. In another method, they consider only the set of images of an object under variable illumination, including multiple, extended light sources, shadows, and color[15,16]. They prove that the set of n-pixel monochrome images of a convex object with a Lambertian reflectance function, illuminated by an arbitrary number of point light sources at infinity, forms a convex polyhedral cone in IR and that the dimension of this illumination cone equals the number of distinct surface normal[17]. Furthermore, the illumination cone can be constructed from as few as three images. In addition, the set of n-pixel images of an object of any shape and with a more general reflectance function, seen under all possible illumination conditions, still forms a convex cone in $\mathrm{IR}_{\mathrm{n}}$. These results immediately suggest certain approaches to 
object recognition. Throughout, they present results demonstrating the illumination cone representation.

\subsection{Support Vector Machine Approach}

Face recognition is a $\mathrm{K}$ class problem, where $\mathrm{K}$ is the number of known individuals; and support vector machines (SVMs) are a binary classification method [18]. By reformulating the face recognition problem and reinterpreting the output of the SVM classifier, they developed a SVM-based face recognition algorithm. The face recognition problem is formulated as a problem in difference space, which models dissimilarities between two facial images. In difference space we formulate face recognition as a two class problem. The classes are: dissimilarities between faces of the same person, and dissimilarities between faces of different people. By modifying the interpretation of the decision surface generated by SVM, we generated a similarity metric between faces that are learned from examples of differences between faces. The SVM-based algorithm is compared with a principal component analysis (PCA) based algorithm on a difficult set of images from the FERET database. Performance was measured for both verification and identification scenarios. The identification performance for SVM is $77-78 \%$ versus $54 \%$ for PCA. For verification, the equal error rate is $7 \%$ for SVM and $13 \%$ for PCA.

\subsection{Karhunen- Loeve Expansion Based Methods}

\subsubsection{Eigen Face Approach}

In this approach, face recognition problem is treated as an intrinsically two dimensional recognition problem [19]. The system works by projecting face images which represents the significant variations among known faces. This significant feature is characterized as the Eigen faces. They are actually the eigenvectors.

Their goal is to develop a computational model of face recognition that is fact, reasonably simple and accurate in constrained environment. Eigen face approach is motivated by the information theory.

\subsubsection{Recognition Using Eigen Features}

While the classical eigenface method uses the KLT (Karhunen- Loeve Transform) coefficients of the template corresponding to the whole face image, the author Pentland et.al. introduce a face detection and recognition system that uses the KLT coefficients of the templates corresponding to the significant facial features like eyes, nose and mouth [20] . For each of the facial features, a feature space is built by selecting the most significant "eigenfeatures", which are the eigenvectors corresponding to the largest eigen values of the features correlation matrix. The significant facial features were detected using the distance from the feature space and selecting the closest match. The scores of similarity between the templates of the test image and the templates of the images in the training set were integrated in a cumulative score that measures the distance between the test image and the training images $[20,16]$. The method was extended to the detection of features under different viewing geometries by using either a view-based Eigen space or a parametric eigenspace.

\subsubsection{Subspace Recognition Approaches}

Principal Component Analysis, LDA, and Bayesian analysis are the three most representative subspace face recognition approaches [21]. In this paper, we show that they can be unified under the same framework. In this approach the author first model face difference with three components: intrinsic difference, transformation difference, and noise. A unified framework is then constructed by using this face difference model and a detailed subspace analysis on the three components. Then they explain the inherent relationship among different subspace methods and their unique contributions to the extraction of discriminating information from the face difference. Based on the framework, a unified subspace analysis method is developed using PCA, Bayes, and LDA as three steps. A 3D parameter space is constructed using the three subspace dimensions as axes. Searching through this parameter space, they achieve better recognition performance than standard subspace methods

\subsubsection{Class Specific Linear Projection Approach}

A face recognition algorithm which is insensitive to lighting direction and facial expression is developed. They adopt the pattern recognition approach for faces in lambertian surface [22]. The method for projection is based on the Fisher's Linear Discriminant and eigen face technique along with correlation and linear subspace. By this they concluded that fisher face method is best at extrapolating and interpolating over variation in lighting.

\subsubsection{Active Pixels Based Approach}

With the recent advances in smart phones and their ease of availability to common man, researchers are exploring efficient algorithms for face recognition on mobile devices for entertainment applications [23]. The limited memory and processing power on mobile devices pose significant challenge to the satisfactory of popular face recognition algorithms like LBP (Local Binary Patterns), Independent Component Analysis, PCA, Neural Networks etc. In this method, the author proposed a novel and efficient algorithm is proposed using Active Pixels which capture the essential local information of the facial image. The brody transform makes the approach more robust to rotational, translational invariance's. The experiments were conducted on standard face recognition databases like FGNET age dataset and color FERET dataset, Texas 3D Face Recognition Database (Texas 3DFRD). The results demonstrated that our approach reduced memory requirement by $80 \%$ and the computational time by $70 \%$ in comparison with LBP approach while yielding same or even better recognition accuracy. 


\subsubsection{Laplacian of Gaussian and Discrete Cosine Transform Based Approach}

The major concerned area of face recognition i.e., single image per person problem where the availability of images is limited to one at training side [24]. To address the problem, the filtration and transforms property of Logarithmic and Discrete Cosine Transform (DCT) to recognize faces are used. As opposed to conventional methods, the idea works at pre-processing stage by filtering images up to four levels and then using the filtered image as an input to DCT for feature extraction using mid frequency values of image. Then, covariance matrix is computed from mean of DCT and Principal component analysis is performed. Finally, distinct feature vector of each image is computed using top Eigenvectors in conjunction with two LOG and DCT images. The experimental comparison for LOG (DCT) was conducted on different standard data sets like ORL, Yale, PIE and MSRA which shows that the proposed technique provides better recognition accuracy than the previous conventional methods of single image per person i.e., (PC) 2A and PCA, 2DPCA, B-2DPCA etc. Hence with over 97\% recognition accuracy, the paper contributes a new enriched feature extraction method at pre-processing

\subsubsection{Kernalised PCA Based Approach}

A B-Spline based kernel principal component analysis (PCA) with doubly nonlinear mapping for human face recognition is implemented [25]. In their approach, the B -Spline wavelets are used to extract facial features, and then a doubly nonlinear mapping kernel PCA (DKPCA) is proposed to perform feature transformation and face recognition. The conventional kernel PCA nonlinearly maps an input image into a high-dimensional feature space in order to make the mapped features linearly separable. In this paper, a new method of nonlinear mapping, which is performed in the original feature space, is defined. The proposed nonlinear mapping not only considers the statistical property of the input features, but also adopts an eigen mask to emphasize those important facial feature points. The new nonlinear mapping is combined with the conventional kernel PCA to be called "doubly" nonlinear mapping kernel PCA. Their work is aimed at comparing the accuracies using Gabor and B-spline wavelets. The same are compared for Gaussian and polynomial kernels.

\subsubsection{PCA on Wavelet Subband Approach}

The new approach in using PCA - apply PCA on wavelet subband. Traditionally, to represent the human face, PCA is performed on the whole facial image [3]. In the proposed method, wavelet transform is used to decompose an image into different frequency subbands, and a mid-range frequency subband is used for PCA representation. In comparison with the traditional use of PCA, the proposed method gives better recognition accuracy and discriminatory power; further, the proposed method reduces the computational load significantly when the image database is large, with more than 256 training images. This paper details the design and implementation of the proposed method, and presents the encouraging experimental results.

\subsubsection{Linear Discriminant Based Method}

The Fisher face method uses the class membership information and develops a set of feature vectors in which variations of different faces are emphasized while different instances of faces due to illumination conditions, facial expressions and orientations are de-emphasized [26]. While the Karhunen-Loeve Transform performs a rotation on a set of axes along which the projection of sample vectors differ most in the autocorrelation sense, the LDT(Linear Discriminant transform) performs a rotation on a set of axes along which the projection of sample vectors differ most in the autocorrelation sense, the LDT performs a rotation on a set of axes along which the projection of sample vectors show maximum discrimination. Each test image is projected onto the optimal LDT space and the resulting set of coefficients is used to compute the Euclidean distance from the images in the training set. Another method of face description for facial image retrieval is from a large data base and for MPEG-7 (Moving Picture Experts Group) standardization [8]. The novel descriptor is obtained by decomposing a face image into several components and then combining the component features [9]. The decomposition combined with LDA (Linear Discriminant Analysis) provides discriminative facial descriptions that are less sensitive to light and pose changes. Each facial component is represented in its Fisher space and another LDA is then applied to compactly combine the features of the components. To enhance retrieval accuracy further, a simple pose classification and transformation technique is performed, followed by recursive matching. Their algorithm has been developed to deal with the problem of face image retrieval from huge databases such as those found in Internet environments. Such retrieval requires a compact face representation which has robust recognition performance under lighting and pose variations. The partitioning of a face image into components offers a number of benefits that facilitate the development of an efficient and robust face retrieval algorithm. Variation in image statistics due to pose and/or illumination changes within each component region can be simplified and more easily captured by a linear encoding than that of the whole image. So an LDA encoding at the component level facilitates better classification. Furthermore, a facial component can be weighted according to its importance. The component with a large variation is weighted less in the matching stage to yield a more reliable decision.

\subsubsection{The "Parametric" Approach Verses The "View-Based Approach}

Murase and Nyar extended the capabilities of the eigenface method to general $3 \mathrm{D}$ object recognition under different illumination and viewing conditions [27]. Given N objects images taken funder $\mathrm{P}$ views and $\mathrm{L}$ different illumination conditions, a set of eigenvectors was obtained 
by applying the eigenface method to all the available data. In this way a single "parametric" describes the object identity as well as the viewing or illumination conditions. The eigenface decomposition of this space was used for feature extraction and classification. However, in order to ensure discrimination between different objects, the number of eigenvectors used in this method was increased compared to the classical eigenface method. The "view based" approach for human face recognition under general viewing condition. The "view based" approach is essentially an extension of the eigenface technique to multiple sets of eigenvectors, one for each face orientation. First, the orientation of the test face is determined by calculating the residual description error (distance from feature space) for each view space, and selecting the sparse for which the distance is minimized. Once the proper view is determined, the face image is classified using the eigenface method in the corresponding space. As expected, the view based representation has better recognition results than the parametric approach, at a cost of a higher computational complexity.

\subsection{Feature Based Methods}

\subsubsection{Kernel Direct Discriminant Analysis Algorithm}

The kernel machine-based Discriminant analysis method deals with the nonlinearity of the face patterns' distribution [1]. This method also effectively solves the so-called "small sample size" (SSS) problem, which exists in most Face Recognition tasks. The new algorithm has been tested, in terms of classification error rate performance, on the multiview UMIST face database. Results indicate that the proposed methodology is able to achieve excellent performance with only a very small set of features being used, and its error rate is approximately $34 \%$ and $48 \%$ of those of two other commonly used kernel FR approaches, the kernel-PCA (KPCA) and the Generalized Discriminant Analysis (GDA), respectively.

\subsubsection{Features Extracted from Walshlet Pyramid}

A novel Walshlet Pyramid based face recognition technique used the image feature set extracted from Walshlets applied on the image at various levels of decomposition [27]. Here the image features are extracted by applying Walshlet Pyramid on gray plane (average of red, green and blue. The proposed technique is tested on two image databases having 100 images each. The results show that Walshlet level-4 outperforms other Walshlets and Walsh Transform, because the higher level Walshlets are giving very coarse color-texture features while the lower level Walshlets are representing very fine color-texture features which are less useful to differentiate the images in face recognition.

\subsubsection{Hybrid Color and Frequency Features Approach}

This correspondence presents a novel hybrid Color and Frequency Features (CFF) method for face recognition [28]. The CFF method, which applies an Enhanced Fisher Model
(EFM), extracts the complementary frequency features in a new hybrid color space for improving face recognition performance. The new color space, the RIQ color space, which combines the component image $\mathrm{R}$ of the $R G B$ color space and the chromatic components I and Q of the $Y I Q$ color space, displays prominent capability for improving face recognition performance due to the complementary characteristics of its component images. The EFM then extracts the complementary features from the real part, the imaginary part, and the magnitude of the $\mathrm{R}$ image in the frequency domain. The complementary features are then fused by means of concatenation at the feature level to derive similarity scores for classification. The complementary feature extraction and feature level fusion procedure applies to the I and Q component images as well. Experiments on the Face Recognition Grand Challenge (FRGC) show that i) the hybrid color space improves face recognition performance significantly, and ii) the complementary color and frequency features further improve face recognition performance.

\subsubsection{Multilevel Block Truncation Coding Approach}

In Multilevel Block Truncation coding for face recognition uses all four levels of Multilevel Block Truncation Coding for feature vector extraction resulting into four variations of proposed face recognition technique [4]. The experimentation has been conducted on two different face databases. The first one is Face Database which has 1000 face images and the second one is "Our Own Database" which has 1600 face images. To measure the performance of the algorithm the False Acceptance Rate (FAR) and Genuine Acceptance Rate (GAR) parameters have been used. The experimental results have shown that the outcome of BTC (Block truncation Coding) Level 4 is better as compared to the other BTC levels in terms of accuracy, at the cost of increased feature vector size.

\subsubsection{Partial Least Square Based Method}

A novel way to perform multi-modal face recognition is to use Partial Least Squares (PLS) to linearly map images in different modalities to a common linear subspace in which they are highly correlated[29]. PLS has been previously used effectively for feature selection in face recognition. They show both theoretically and experimentally that PLS can be used effectively across modalities. They also formulate a generic intermediate subspace comparison framework for multi-modal recognition. Surprisingly, they achieve high performance using only pixel intensities as features. They experimentally demonstrate the highest published recognition rates on the pose variations in the PIE data set, and also show that PLS can be used to compare sketches to photos, and to compare images taken at different resolutions. Their approach matches probe and gallery images by linearly projecting them into an intermediate space where images with the same identity are highly correlated. They argue that for a variety of cross-modality recognition problems, such projections will exist and can be found using PLS and BLM. 


\subsubsection{Multi-Resolution Local Appearance Approach}

Facial analysis based on local regions / blocks usually outperforms holistic approaches because it is less sensitive to local deformations and occlusions [9]. Moreover, modeling local features enables us to avoid the problem of high dimensionality of feature space. In this paper, the author model the local face blocks with Gabor features and project them into a discriminant identity space. The similarity score of a face pair is determined by fusion of the local classifiers. To acquire complementary information in different scales of face images, the local decisions were integrated from various image resolutions. They proposed multi-resolution block based face verification system which is evaluated on the experiment of Face Recognition Grand Challenge (FRGC) version 2.0. They obtained $92.5 \%$ verification rate and $1 \%$ false acceptance rate.

In another work, the author extended recognition to uncontrolled situations is a key challenge for practical face recognition systems [30]. Finding efficient and discriminative facial appearance descriptors is crucial for this. Most existing approaches use features of just one type. Here they argue that robust recognition requires several different kinds of appearance information to be taken into account, suggesting the use of heterogeneous feature sets. They show that combining two of the most successful local face representations, Gabor wavelets and Local Binary Patterns (LBP), gives considerably better performance than either alone: they are complimentary in the sense that LBP captures small appearance details while Gabor features encode facial shape over a broader range of scales. Both feature sets are high dimensional so it is beneficial to use PCA to reduce the dimensionality prior to normalization and integration. The Kernel Discriminative Common Vector method is then applied to the combined feature vector to extract discriminant nonlinear features for recognition. The method is evaluated on several challenging face datasets including FRGC 1.0.4, FRGC 2.0.4 and FERET, with promising results

\subsection{Neural Network Based Algorithms}

Templates have been also used as input to Neural Network (NN) based systems. Lawrence et.al proposed a hybrid neural network approach that combines local image sampling, A self organizing map (SOM) and a convolutional neural network [31]. The SOP provides a set of features that represents a more compact and robust representation of the image samples. These features are then fed into the convolutional neural network. This architecture provides partial invariance to translation, rotation, scale and face deformation. Along with this the author introduced an efficient probabilistic decision based neural network (PDBNN) for face detection and recognition. The feature vector used consists of intensity and edge values obtained from the facial region of the down sampled image in the training set. The facial region contains the eyes and nose, but excludes the hair and mouth. Two PDBNN were trained with these feature vectors and used one for the face detection and other for the face recognition.

\subsubsection{Training Feed forward Networks with the Marquardt Algorithm}

The presented Marquardt algorithm for nonlinear least squares and is incorporated into the back propagation algorithm for training feed forward neural networks. The algorithm is tested on several function approximation problems, and is compared with a conjugate gradient algorithm and a variable learning rate algorithm [32]. It is found that the Marquardt algorithm is much more efficient than either of the other techniques when the network contains no more than a few hundred weights

\subsubsection{Transformation Invariance in Pattern Recognition}

In pattern recognition, statistical modeling, or regression, the amount of data is a critical factor affecting the performance. If the amount of data and computational resources are unlimited, even trivial algorithms will converge to the optimal solution [33]. However, in the practical case, given limited data and other resources, satisfactory performance requires sophisticated methods to regularize the problem by introducing a priori knowledge. Invariance of the output with respect to certain transformations of the input is a typical example of such a priori knowledge. The author introduced the concept of tangent vectors, which compactly represent the essence of these transformation invariance, and two classes of algorithms, tangent distance and tangent propagation, which make use of these invariance to improve performance.

\subsubsection{Face Recognition Technology}

Several famous face recognition algorithms, such as eigenfaces and neural networks, were also being explained [34]. In order to cope with complication in the task of face recognition variations and find out the true invariant for recognition, researchers have developed various recognition algorithms. In this section, they described two representative ones. The eigenface approach applies the Karhonen-Loeve (KL) transform for feature extraction. It greatly reduces the facial feature dimension and yet maintains reasonable discriminating power. The neural network approach, though some variants of the algorithm work on feature extraction as well, mainly provides sophisticated modeling scheme for estimating likelihood densities in the pattern recognition phase. Probabilistic Decision- based Neural Network (PDNN) does not have the fully connected network topology. Instead, it divides the network into $\mathrm{K}$ subnets. Each subnet is dedicated to recognize one person in the database. PDNN uses the Gaussian activation function for its neurons, and the output of each "face subnet" is the weighted summation of the neuron outputs.

\subsubsection{Fuzzy Hybrid Learning Algorithm}

The fuzzy hybrid learning algorithm (FHLA) for the radial basis function neural network (RBFNN) determines the 
number of hidden neurons in the RBFNN structure by using cluster validity indices with majority rule while the characteristics of the hidden neurons are initialized based on advanced fuzzy clustering [35]. The FHLA combines the gradient method and the linear least-squared method for adjusting the RBF parameters and the neural network connection weights. The RBFNN with the proposed FHLA is used as a classifier in a face recognition system. The inputs to the RBFNN are the feature vectors obtained by combining shape information and principal component analysis. The designed RBFNN with the proposed FHLA, while providing a faster convergence in the training phase, requires a hidden layer with fewer neurons and less sensitivity to the training and testing patterns. The efficiency of the proposed method is demonstrated on the ORL and Yale face databases, and comparison with other algorithms indicates that the FHLA yields excellent recognition rate in human face recognition.

\subsubsection{Neural Networks using Skin Color Segmentation}

A robust schema for face detection system via Gaussian mixture model to segment image based on skin color is chosen. After skin and non skin face candidates' selection, features are extracted directly from discrete cosine transform (DCT) coefficients computed from these candidates. Moreover, the back-propagation neural networks are used to train and classify faces based on DCT feature coefficients in $\mathrm{Cb}$ and $\mathrm{Cr}$ color spaces [36]. This schema utilizes the skin color information, which is the main feature of face detection. DCT feature values of faces, representing the data set of skin/non-skin face candidates obtained from Gaussian mixture model are fed into the back-propagation neural networks to classify whether the original image includes a face or not. Experimental results shows that the proposed schema is reliable for face detection and pattern features are detected and classified accurately by the back propagation neural networks.

\subsubsection{Detection using Polynomial Neural Network}

A new face detection method using a polynomial neural network (PNN) locates the human faces in an image, the local regions in multiscale sliding windows are classified by the PNN to two classes, namely, face and non-face [37]. The PNN takes as inputs the binomials of the projection of local image onto a feature subspace learned by principal component analysis (PCA). They investigated the influence of PCA on either the face samples or the pooled face and non-face samples. In addition, the distance was integrated from the feature subspace into the PNN to improve the detection performance. In experiments on images with complex backgrounds, the proposed method has produced promising results in terms of high detection rate and low false positive rate.

\subsubsection{Detection Using Information Fusion}

The fundamental point is that the fusion of several simple, somewhat unreliable, and somewhat inefficient frontal face detectors results in an efficient and reliable frontal face detector which, without any training, performs similarly to a state-of the- art neural network based face detector trained on 60,000 images[38]. In this paper, they proposed several extremely simple face detection approaches. These approaches are not based on any prior training, but are instead based on common sense metrics. It is shown that the combination or fusion of these approaches results in a relatively accurate, face, and reliable face detector which outperforms all of the individual detectors and, without any training, performs similarly to state-of-the-art face detectors which require extensive training.

\subsubsection{Detection and Localization of Faces Using MLP}

Automatic detection and localization of faces on digital images is based on learning by example and multi-resolution analysis of digital images [39]. The objective of detection and localization of faces is to provide the coordinates of the frames including the faces in an image. The approach is based on machine learning. The advantage of such an approach is the possibility to adapt to any special condition (people, environment), and to improve the performances by increasing the size of the data-base. Special emphasis is put on the management of the learning data, in order to improve the performances. Various experimental results, obtained by using a Multi-Layer Perceptron (MLP) as a classifier, are provided.

\subsubsection{Depth and Curvature Feature Based Recognition}

This method explored face recognition from a representation based on features extracted from range images [40]. Depth and curvature features have several advantages over more traditional intensity based features. Specifically, curvature descriptors 1) have the potential for higher accuracy in describing surface based events, 2) are better suited to describe properties of the face in areas such as the cheeks, forehead, and chin, and 3) are viewpoint invariant. Faces are represented in terms of a vector of feature descriptors. Comparison between two faces is made based on their relationship in the feature space. They provided detailed analysis of the accuracy and discrimination of the particular features extracted, and of the effectiveness of the recognition system for the test database of 24 faces. Results are very promising. In many cases it is shown that feature accuracy is limited more by surface resolution than by the extraction process.

\subsubsection{Euclidean Distance of Images}

Euclidean distance for images, which they call IMage Euclidean Distance (IMED), is robust to small perturbation of images [41]. IMED is then applied to image recognition. The key advantage of this distance measure is that it can be embedded in most image classification techniques such as SVM, LDA and PCA. The embedding is rather efficient by involving a transformation referred to as Standardizing Transform (ST). They show that ST is a transform domain smoothing. Using the Face Recognition Technology 
(FERET) database and two state-of-the-art face identification algorithms, they demonstrate a consistent performance improvement of the algorithms embedded with the new metric over their original versions.

\subsection{Model Based Methods}

\subsubsection{Hidden Markov Model Based Approach}

In this approach, the author utilizes the face that the most significant facial features of a frontal face which includes hair, forehead, eyes, nose and mouth which occur in a natural order from top to bottom even if the image undergo small variation/rotation in the image plane perpendicular to the image plane [42]. One dimensional HMM (Hidden Markov Model) is used for modeling the image, where the observation vectors are obtained from DCT or KLT coefficients. They given c face images for each subject of the training set, the goal of the training set is to optimize the parameters of the Hidden Markov Model to best describe the observations in the sense of maximizing the probability of the observations given in the model. Recognition is carried out by matching the best test image against each of the trained models. To do this, the image is converted to an observation sequence and then model likelihoods are computed for each face model. The model with the highest likelihood reveals the identity of the unknown face.

\subsubsection{The Volumetric Frequency Representation of Face Model}

A face model that incorporates both the three dimensional (3D) face structure and its two-dimensional representation are explained (face images) [43]. This model which represents a volumetric (3D) frequency representation (VFR) of the face, is constructed using range image of a human head. Making use of an extension of the projection Slice Theorem, the Fourier transform of any face image corresponds to a slice in the face VFR. For both pose estimation and face recognition a face image is indexed in the 3D VFR based on the correlation matching in a four dimensional Fourier space, parameterized over the elevation, azimuth, rotation in the image plane and the scale of faces.

\subsubsection{User Oriented Model Based Approach}

It is a novel approach for a user oriented language model for face detection [19]. Even though there is many open source or commercial libraries to solve the problem of face detection, they are still hard to use because they require specific knowledge on details of algorithmic techniques. The author proposed a high-level language model for face detection with which users can develop systems easily and even without specific knowledge on face detection theories and algorithms. Important conditions are firstly considered to categorize the large problem space of face detection. The conditions identified here are then represented as expressions in terms of a language model so that developers can use them to express various problems. Once the conditions are expressed by users, the proposed associated interpreter interprets the conditions to find and organize the best algorithms to solve the represented problem with corresponding conditions. It showed a proof-of-concept implementation and some test and analyze example problems to show the ease of use and usability.

\subsubsection{Multiview Recognition Approach}

This method is to recognize a multiview face (i.e., to recognize a face under different views) through optimization of multiple single-view face recognitions [26]. Many face descriptors show quite satisfactory results to recognize identity of people with given limited view (especially for the frontal view), but the full view of the human head has not yet been recognizable with commercially acceptable accuracy. As there are various single-view recognition techniques already developed for very high success rate, for instance, MPEG-7 advanced face recognizer, they propose a new paradigm to facilitate multiview face recognition, not through a multiview face recognizer, but through multiple single-view recognizers. To retrieve faces in any view from a registered descriptor, they give corresponding view information to the descriptor. As the descriptor needs to provide any requested view in 3D space, it is referred as "3D" information that it needs to contain. Their analysis in various angled views checks the extent of each view influence and it provides a way to recognize a face through optimized integration of single view descriptors covering the view plane of horizontal rotation and vertical rotation. The resulting face descriptor based on multiple representative views, which is of compact size, shows reasonable face recognition performance on any view. Hence, their face descriptor contains quite enough $3 \mathrm{D}$ information of a person's face to help for recognition and eventually for search, retrieval, and browsing of photographs, videos, and 3D-facial model databases.

\section{Conclusion}

This paper discusses the different approaches which have been employed in automatic face recognition. In the geometrical based methods, the geometrical features are selected and the significant facial features are detected. The correlation based approach needs face template rather than the significant facial features. Singular value vectors and the properties of the SV vector provide the theoretical basis for using singular values as image features. The Karhunen-Loeve expansion works by projecting the face images which represents the significant variations among the known faces. Eigen values and Eigen vectors are involved in extracting the features in KLT. Neural network based approaches are more efficient when it contains no more than a few hundred weights. The Hidden Markov model optimizes the parameters to best describe the observations in the sense of maximizing the probability of observations given in the model . Some methods use the features for 
classification and few methods uses the distance measure from the nodal points. The drawbacks of the methods are also discussed based on the performance of the algorithms used in the approaches. Hence this will give some idea about the existing methods for automatic face recognition.

\section{REFERENCES}

[1] Anastasios N. Venetsanopoulos “ Face Recognition Using Kernel Direct Discriminant Analysis Algorithms";IEEE Transactions On Neural Networks, Vol. 14, No. 1, pp.117-126,2003.

[2] R.Chellappa.C. Wilson and S.Sirohey; "Human and Machine recognition of face", Proceedings of IEEE, vol 83,pp.705-741,1995.

[3] Ara V.Nefian, And Monsoon H.Hayes, “ Hidden Markov Models For Face Detection And Recognition",IEEE Transcations On Pattern Analysis And Machine Intelligence, Vol.1,pp.141-145,1999.

[4] Dr. H.B.Kekre, Sudeep D. Thepade , Akshay Maloo , MPSTME(2011); "Face Recognition Using Texture Feartures Extracted Form Walshlet Pyramid",Int. J. on Recent Trends in Engineering \& Technology, Vol. 05, No. 0,pp.186,2011.

[5] Dr.H.B.Kekre, Dr.Sudeep, D.Thepade, Sanchit Khandelwal, "Face Recognition Using Multilevel Block Truncation Coding"; International Journal of Computer Applications () Volume 36- No.11,pp.0975 - 8887,2011.

[6] Anastasios N. Venetsanopoulos, “ Face Recognition Using Kernel Direct Discriminant Analysis Algorithms";IEEE Transactions On Neural Networks, Vol. 14, No. 1 ,pp.118-126,2003.

[7] M.LADES, J. Buhman ,J.Lange,C.Malsburg And R.Wurtz, "Distortion Invariant Object Recognition In The Dynamic Link Architecture,"; IEEE Transactions On Computers, Vol. 42,No.3,pp .300-311,1993.

[8] Philips, "Matching Pursuits Filter Design applied to Face Recognition", ARL-TR-1487, Army research laboratory,USA, 1998.

[9] Philips, "Matching Pursuit Filters Design",Proceedings of the 12th International Conference On Pattern Recognition,Vol.3,Pp 57-61,1994.

[10] Z.Hong;"Algebraic Feature Extraction Of Image For Recognition, "Pattern Recognition, Vol 24, No.3, pp. 211-219, 1991.

[11] R.Brunelli and T.Poggio,face recognition(1993); "Features verses templates",IEEE Transcations on pattern analysis and Machine Intelligence,,Vol 15, pp.1042 - 1052,1993.

[12] Sangeeta N. Kakarwal, Ratnadeep R. Deshmukh, , "Hybrid Feature Extraction Technique for Face Recognition"; (IJACSA) International Journal of Advanced Computer Science and Applications, Vol. 3, No.2,pp.60-64,2012.

[13] Q Tian; “Comparison Of Statistical Pattern Recognition Algorithms For Hybrid Proceedings,Ii:Eigen Vector Based Algorithms,"Journal Of The Optimal Society Of
America,Vol. 5,Pp.1670-1672,1988.

[14] R. Ramamoorthi and P. Hanrahan, "On the relationship between radiance and irradiance: Determining the illumination from images of a convex Lambertian object," J. Opt. Soc. Amer., vol. 18, no. 10, pp. 2448-2459,2001.

[15] Hansen F. Chen, Peter N. Belhumeur, David W. Jacobs, "In Search of Illumination Invariants"; IEEE transcations on pattern recognition, Vol 9, 2000.

[16] P. N. Belhumeur and D. J. Kriegman "What is the set of images of an object under all possible lighting conditions?," in Proc. IEE Conf. Comput. Vis. Pattern Recognition, Vol.28,issue 3, pp. 1-16,1998.

[17] R. Gross and V. Brajovie, "An image preprocessing algorithm for illumination Invariant face recognition," in Proc. 4th Int. Conf. Audio Video Based Biometric Person Authentication, Vol. 2688/, pp. 10-18, 2003.

[18] Juwei Lu, Konstantinos N. Plataniotis, L.D Harmon, M.K.Khan,m R.Lasch And P.F.Ramig."Machine Identification Of Human Faces, "Pattern Recognition,Vol.13,issue2,pp.97-110,1981.

[19] M. Daesik Jang; "User Oriented Language Model for Face Detection"; IEEE workshop on person oriented vision,pp.21-26,2011.

[20] Mathew Turk And Alex Pentland, “ Eigen Faces For Recognition"; Journal Of Cognitive Neuroscience Vol.3,No.1,pp.71-86,1991.

[21] Xiaogang Wang and Xiaoou Tang, "A Unified Framework for Subspace Face Recognition";IEEE Transactions On Pattern Analysis And Machine Intelligence, Vol. 26, No. 9,pp. $1222-1228,2004$.

[22] A.J Goldstein L.Harmon And A.Lesk,. “ Identification Of Human Faces',Proceedings Of The IEEE,,Vol59,issue 5,pp. $748-760,1971$

[23] Mallikarjuna Rao G, Praveen Kumar, Vijaya Kumari G, Amit Pande, Babu G.R, "Role Of Active Pixels For Efficient Face Recognition On Mobile Environment" International Journal of Intelligent Information Processing(IJIIP) Volume2, Number3,2011.

[24] W. Yin, and Z. Deng, "Image-based face illumination transferring using logarithmic total variation models," Int. Journal of. Computer. Graph., Vol. 26, no. 1, pp. 41-49,2009.

[25] P. N. Belhumeur, J. P. Hespanha, and D. J. Kriegman, "Eigenface vs. fisherfaces: Recognition using class specific linear projection," IEEE Trans. Pattern Anal. Machine Intelligence., vol. 19, no. 7, pp. 711-720, 1997.

[26] Dr. H.B.Kekre, Sudeep D. Thepade, Akshay Maloo, "Face Recognition Using Texture Feartures Extracted Form Walshlet Pyramid",Int. J. on Recent Trends in Engineering \& Technology, Vol. 05, No. 01,2011.

[27] P. Jonathon Phillips, (1999). ;“ Support Vector Machines applied to face Recognition" Proceedings of the ICWET '11 International Conference \& Workshop on Emerging Trends in Technology,pp.803-809,2011.

[28] Abhishek Sharma;" bypassing synthesis: PLS for face recognition"; Institute of Advanced computer studies, uni,of merryland USA,pp.593-600,2011. 
[29] Xiaoyang Tan and Bill Triggs, "Fusing Gabor and LBP Feature Sets for Kernel-based Face Recognition"3rd International Workshop Analysis and Modelling of Faces and Gestures (AMFG '07) 4778, 2007.

[30] Y Cheng,K.Liu.J.Jang,Y.Zhang,And N.Gu, "Human Face Recognition Method Based On The Statistical Model Of Small Sample Size",In SPIE Proceedings Intelligent Robots And Computer Vision X:ALG AND Techn, Vol 1607,pp.85-95,1991.

[31] Martin T. Hagan and Mohammad B. Menhaj, "Training Feedforward Networks with the Marquardt Algorithm" IEEE Transactions On Neural Networks, VOL. 5, NO. 6, pp.986-993, 1994.

[32] Yann A. Le Cun ,Patrice Y. Simard, John S. Denker, Bernard Victorri," Transformation Invariance in Pattern Recognition: Tangent Distance and Propagation", Neural Networks: Tricks of the Trade, Springer-Verlag, Berlin, pp 239-274, 1998.

[33] Shang-Hung Lin, Ph.D.” An Introduction to Face Recognition Technology", informing science special issue on multimedia informing technologies-part 2-vol 3 no 1 , pp.1-7,2000.

[34] Majid Ahmadi ,Javad Haddadniaa, Karim Faeza, ," A fuzzy hybrid learning algorithm for radial basis function neural network with application in human face recognition", Pattern Recognition Society,Vol.36,issue.5,pp.1187-1202,2003.

[35] Ying Weng, Aamer Mohamed, Jianmin Jiang and Stan Ipson," Face Detection based Neural Networks using Robust Skin
Color Segmentation", 2008 5th International Multi-Conference on Systems, Signals and Devices,pp.1-5,2008.

[36] Yoshihiro Hagihara ,Lin-Lin Huang, Akinobu Shimizu, , "Face detection from cluttered images using a polynomial neural network", Neuro computing Elsevier Science ,pp.197-211,2003.

[37] Parham Aarabi, Jerry Chi Ling Lam, Arezou Keshavarz," Face Detection Using Information Fusion"; 11th conference on machine learning,pp1-8,2007.

[38] Gilles BUREL \& Dominique CAREL," Detection and localization of faces on digital images", Pattern Recognition Letters, Vol. 15, no 10, pp.1-9, 1994.

[39] Gaile G. Gordon," Face Recognition Based on Depth and Curvature Features"; International journal of pattern recognition,vol 16,pp808-810,1992.

[40] Yan Zhang ,Liwei Wang, Jufu Feng," On the Euclidean Distance of Images", Center for Information Sciences, Vol.27,issue.8,pp.1334-1339,2005.

[41] D. J. Jobson, Z. Rahman, and G. A. Woodell, "Properties and performance of face Recognition"; IEEE Transactions on Image Processing, Vol.6,issue.3,pp.451-462, 1997.

[42] Mallikarjuna Rao G, Praveen Kumar, Vijaya Kumari G, Amit Pande, Babu G.R; (2011) “ Role Of Active Pixels For Efficient Face Recognition On Mobile Environment" International Journal of Intelligent Information Processing(IJIIP) Volume2, Number3,pp.1-7,2013. 